\title{
Hipotiroidismo Subclínico en Pacientes con Obesidad y Sobrepeso
}

\author{
a Centro de Salud de \\ Camarena (Toledo). \\ ' Centro de Salud \\ "Palomarejos" de Toledo. \\ "Centro de Salud de \\ Menasalbas (Toledo). \\ d Unidad Docente de Medicina \\ de Familia de Toledo.
}

Correspondencia:

Raúl Calvo Rico, Centro de

Salud de Camarena,

c/ José Antonio, $n^{\circ} 1$,

Camarena, 45180 - Toledo

(España). Correo electrónico:

rcalvor@sescam.jccm.es

Recibido el 10 de septiembre de 2010.

Aceptado para su publicación el 25 de septiembre de 2010.

\section{RESUMEN}

Objetivo. Existen dudas sobre la posible influencia de la obesidad en la función tiroidea y su relación con el hipotiroidismo subclínico (HS). El objetivo de este trabajo fue conocer la prevalencia de HS en personas con un índice de masa corporal (IMC) $\geq 25 \mathrm{~kg} / \mathrm{m}^{2}$.

Material y métodos. Estudio observacional descriptivo, realizado en mayores de 18 años, pertenecientes al área de salud de Toledo. La muestra se obtuvo mediante muestreo consecutivo en consulta. A cada persona se le realizó un estudio analítico (TSH y T4 libre) y se calculó su IMC, registrando su edad, sexo, hábito tabáquico, antecedentes de patología tiroidea, hábitat y nacionalidad. Se consideró HS si TSH $>4 \mu \mathrm{U} / \mathrm{ml}$ y T4 libre $>0,8 \mathrm{ng} / \mathrm{dl}$, según el rango de valores establecidos por el laboratorio de referencia.

Resultados. La muestra la formaron 332 personas (53,9\% mujeres, edad media 59,5 años). Eran fumadores el $12,9 \%$ y tenían antecedentes de patología tiroidea el 7,3\%. El $52,4 \%$ eran obesos y el $47,6 \%$ presentaba sobrepeso. La prevalencia de HS fue de $9,3 \%$ (IC 95\%: 6,5-13,1). No observamos asociación significativa entre HS y edad, tabaquismo o hábitat. Tampoco encontramos correlación entre IMC y TSH (rho $=-0,033 ; p=0,546)$. La frecuencia de HS en mujeres $(13,4 \%)$ fue mayor que en hombres $(4,6 \%)(p=0,006)$.

Conclusiones. La prevalencia de HS en población con exceso de peso no difiere de las halladas en otros estudios realizados en población general. No hemos encontrado correlación entre los niveles de IMC y los valores séricos de TSH. El HS es más frecuente en mujeres, pero no hemos hallado asociación con la edad o el hábito tabáquico.

Palabras clave: Obesidad, Sobrepeso, Índice de Masa Corporal, Hipotiroidismo subclínico.

\section{ABSTRACT}

\section{Sub-clinical hypothyroidism in obese and overweight patients}

Objective. The possible influence of obesity on thyroid function and its relationship with subclinical hypothyroidism $(\mathrm{SH})$ is uncertain. The objective of this study was to determine the prevalence of $\mathrm{SH}$ in people with a BMI $\geq 25 \mathrm{~kg} / \mathrm{m}^{2}$.

Materials and methods. Descriptive, observational study, conducted in individuals over 18 years, in the Toledo health area. The sample was obtained through consecutive sampling in the doctor's consultancy. Thyroid tests were conducted on each person (TSH, free T4) and their BMI was calculated. Their age, sex, smoking habit, history of thyroid disease, environment, and nationality were recorded. Diagnosis of $\mathrm{SH}$ was considered as a TSH level of $>4 \mu \mathrm{U} / \mathrm{ml}$ and a free T4 level of $>0.8 \mathrm{ng} / \mathrm{dl}$, according to the laboratory reference range. .

Results. The sample comprised 332 persons; $53.9 \%$ female; mean age, 59.5 years. Twelve point nine percent were smokers and $7.3 \%$ had a history of thyroid disease; $52.4 \%$ were obese and $47.6 \%$ overweight. The prevalence of $\mathrm{SH}$ was $9.3 \%$ (95\% Cl 6.5-13.1). We found no significant relationship between $\mathrm{SH}$ and age, smoking, or environment. We found no correlation between BMI and TSH (rho $=-0.033 ; p=0.546)$. The frequency of SH in women $(13.4 \%)$ was higher than in men $(4.6 \%)$ [ $p=0.006]$.

Conclusions. SH prevalence in an overweight population does not differ from those found in other studies in the general population. We did not find a correlation between BMI and serum TSH levels. SH is more common in women, but we did not find any relationship with age or smoking habit.

Keywords: Obesity, Overweight, Body mass index, Subclinical hypothyroidism. 


\section{INTRODUCCIÓN}

La ganancia ponderal ha sido incluida clásicamente como uno de los síntomas clínicos del déficit de hormonas tiroideas; de hecho, varios estudios han relacionado el aumento sérico de TSH con el incremento del índice de masa corporal ${ }^{1-3}$, e incluso se ha demostrado una correlación entre los niveles de TSH y el porcentaje de grasa corporal ${ }^{4}$.

Sin embargo, no está tan clara la influencia que puede tener el aumento de peso en la función tiroidea. Algunos estudios sugieren que la obesidad puede derivar en una hipofunción tiroidea ${ }^{5,6}$, pero existen resultados contradictorios. Dada la elevada prevalencia en nuestra sociedad de obesidad (en nuestro país, casi el $17 \%$ de los adultos ${ }^{7}$, pero en EEUU se habla de prevalencias de hasta un $32,2 \%$ en varones y $35,5 \%$ en mujeres ${ }^{8}$ ), de ser cierta esta relación entre aumento de peso y niveles de $\mathrm{TSH}$, gran parte de nuestra población con exceso de peso debería tener una disfunción tiroidea, presumiblemente subclínica.

El hipotiroidismo subclínico es una condición caracterizada por niveles de hormona tiroidea periféricos dentro del rango de referencia normal, pero una cifra de TSH ligeramente elevada ${ }^{9}$. Según algunas revisiones recientes, está presente en el 3,0-8,5\% de la población general, es más común en mujeres y su prevalencia aumenta con la edad ${ }^{10}$.

Aunque existen muchos estudios sobre la prevalencia de hipotiroidismo, clínico o subclínico, en población general, hay pocos datos en personas con sobrepeso u obesidad. En este contexto, nos propusimos estudiar la prevalencia de hipotiroidismo subclínico en personas con un índice de masa corporal (IMC) mayor o igual de $25 \mathrm{~kg} / \mathrm{m}^{2}$. Secundariamente, quisimos conocer si existía correlación estadística entre el IMC y el valor de TSH sérico en estos pacientes y explorar la posible asociación de hipotiroidismo subclínico a otros factores como la edad, el sexo o el hábito tabáquico.

\section{MATERIAL Y MÉTODO}

Se trata de un estudio observacional descriptivo, realizado en pacientes mayores de 18 años, con IMC mayor de $25 \mathrm{~kg} / \mathrm{m}^{2}$, pertenecientes a las zonas básicas de salud de Palomarejos, Menasalbas y Camarena, todas pertenecientes al área de salud de Toledo y con una población total aproximada de 30.000 habitantes. Se excluyeron del estudio gestantes y pacientes en tratamiento con hormonas tiroideas.

La muestra se obtuvo mediante muestreo consecutivo (reclutamiento) de pacientes consultantes (por cualquier causa) en cinco cupos de Medicina, tres urbanos y dos rurales. El tamaño muestral necesario para estimar la prevalencia de hipotiroidismo en obesos / sobrepesos con una frecuencia esperada del $10 \%$, una precisión de $\pm 4 \%$ y $p<0,05$ fue de 315 individuos.

A cada persona captada, previa información de los objetivos del estudio, se le ofertó la realización de un estudio analítico que incluía niveles séricos de TSH y T4 libre. Las determinaciones fueron realizadas en el laboratorio de referencia habitual (Complejo Hospitalario de Toledo). Se les realizó también un tallaje y pesaje con tallímetros y básculas de las consultas, calculándose el IMC mediante la fórmula Peso $(\mathrm{kg}) /$ Talla $(\mathrm{m})^{2}$ y etiquetándoles en función del mismo como sobrepeso (IMC $\geq 25$ ) u obesidad ( $\geq 30)$. Se consideró válido para el estudio a los pacientes que tenían registrados en su historia los valores de TSH y T4 libres, así como el IMC durante el año 2009 , siempre y cuando no existiese un lapso de tiempo superior a los 2 meses entre la fecha de la analítica y la del IMC y no se hubiese producido una pérdida de peso significativa desde la recogida de dichos datos hasta su inclusión en el estudio.

Junto a los valores hormonales referidos y el IMC, se registraron también las siguientes variables: edad, sexo, hábito tabáquico (fumador, no fumador o exfumador), antecedentes personales de patología tiroidea (según diagnóstico registrado en su historia clínica electrónica), procedencia de medio rural (< 5.000 habitantes) o urbano, y nacionalidad.

Se consideró hipotiroidismo subclínico cuando el valor de la TSH sobrepasaba los $4 \mu \mathrm{U} / \mathrm{ml}$ con cifras de T4 libre superiores a 0,8 ng/dl, según el rango de valores establecidos por el citado laboratorio de referencia.

Los datos obtenidos fueron introducidos en una base del programa estadístico SPSS 12.0 para Windows. Para su descripción se emplearon medias y porcentajes, calculándose el intervalo de confianza del $95 \%$ para los resultados principales. Las pruebas de análisis utilizadas fueron la $\chi^{2}$ de Pearson, la $U$ de Mann-Whitney y el coeficiente de correlación $\rho$ (rho) de Spearman, previa confirmación de la no-normalidad de las variables estudiadas. Se realizó una regresión logística binaria para valorar los posibles factores asociados a la presencia de hipotiroidismo.

\section{RESULTADOS}

La muestra final la formaron 332 personas, con un porcentaje de mujeres del $53,9 \%$ y una edad media de 59,5 años (DE: 15,5). Procedía del medio rural 


\begin{tabular}{|c|c|c|}
\hline Variable & Hipotiroidismo (\%) & Significación estadística \\
\hline $\begin{aligned} \text { Sexo } & \\
- & \text { Hombres } \\
- & \text { Mujeres }\end{aligned}$ & $\begin{array}{c}4,6 \\
13,4\end{array}$ & $\begin{array}{c}\chi^{2}=7,602 \\
p=0,006\end{array}$ \\
\hline $\begin{array}{cc}\text { Grupos de Edad } \\
-\quad 18-45 \text { años } \\
-\quad 46-65 \text { años } \\
-\quad 65 \text { años }\end{array}$ & $\begin{array}{c}11,1 \\
7,6 \\
10,5\end{array}$ & $\begin{array}{l}\chi^{2}=0,952 \\
p=0,621\end{array}$ \\
\hline $\begin{array}{cl}\text { Procedencia } \\
- & \text { Urbana } \\
- & \text { Rural }\end{array}$ & $\begin{array}{c}10,6 \\
7,5\end{array}$ & $\begin{array}{l}\chi^{2}=0,867 \\
p=0,352\end{array}$ \\
\hline $\begin{aligned} & \text { Hábito tabáquico } \\
&- \text { Fumador } \\
&- \text { No fumador * }\end{aligned}$ & $\begin{array}{l}9,3 \\
9,3\end{array}$ & $\begin{array}{l}\chi^{2}=0,000 \\
p=0,997\end{array}$ \\
\hline $\begin{array}{cc}\text { Grado de } & \text { sobrepeso } \\
- & \text { Sobrepeso } \\
- & \text { Obesidad }\end{array}$ & $\begin{array}{l}9,8 \\
8,8\end{array}$ & $\begin{array}{c}\chi^{2}=0,102 \\
p=0,749\end{array}$ \\
\hline
\end{tabular}

Tabla 1. Frecuencia de hipotiroidismo subclínico según sexo, edad, procedencia, hábito tabáquico y grado de sobrepeso.

* Incluye exfumadores por necesidades del análisis estadístico.

\begin{tabular}{l|cccccc}
\hline & & & & \multicolumn{2}{c}{ Odds } & \multicolumn{2}{c}{ IC $_{95 \%}$ Odds Ratio } \\
Covariables & B & Wald & Sig. & Ratio & Inferior & Superior \\
\hline Sexo (mujer/hombre) & 1,208 & 7,142 &, 008 & 3,347 & 1,380 & 8,117 \\
Edad &, 001 &, 002 &, 961 & 1,001 &, 976 & 1,026 \\
IMC &,- 005 &, 013 &, 909 &, 995 &, 906 & 1,091 \\
Hábitat &, 342 &, 630 &, 428 & 1,408 &, 605 & 3,277 \\
Tabaquismo &,- 241 &, 146 &, 702 &, 786 &, 229 & 2,699 \\
Constante & $-2,912$ & 2,857 &, 091 &, 054 & & \\
\hline
\end{tabular}

Tabla 2. Modelo de regresión logística binaria (método Enter). Variable dependiente: hipotiroidismo subclínico.

el $40,1 \%$. El $95,2 \%$ de los individuos de la muestra eran de nacionalidad española.

Eran fumadores el $12,9 \%$ y exfumadores el $10,8 \%$. Contaban con antecedentes de patología tiroidea el $7,3 \%$, de los cuales un $45,8 \%$ era hipotiroidismo subclínico, un $29,1 \%$ nódulos tiroideos, y un $25,1 \%$ otras patologías tiroideas. El IMC medio era de 30,5 (DE: 4.4), y la distribución entre obesos y sobrepesos era de $52,4 \%$ y $47,6 \%$ respectivamente. El valor medio de TSH era de $2,2 \mu \mathrm{U} / \mathrm{ml}(\mathrm{DE}: 1,8)$ y el de T4 de 1,2 ng/dl (DE: 0,4).

La prevalencia de hipotiroidismo subclínico hallada en nuestra muestra de obesos y sobrepesos fue de 9,3\% (IC 95\%: 6,5-13,1). No se observó asociación significativa entre la existencia de hipotiroidismo subclínico y la edad, el hábito tabáquico y el hábitat (tabla 1). Sin embargo, los niveles de TSH sérico registrados fueron mayores entre la población de procedencia urbana $(2,41 \mu \mathrm{U} / \mathrm{ml})$ que entre la población de procedencia rural $(2,03)$, siendo esta diferencia estadísticamente significativa $\left(\mathrm{U}_{\mathrm{MW}}=\right.$ 10276; $p=0,001)$.

No encontramos correlación entre IMC y TSH $(\rho=$ $-0,033 ; p=0,546)$ ni entre edad y TSH $(\rho=-0,026$; $p=0,631)$

La frecuencia de hipotiroidismo subclínico en mujeres $(13,4 \%)$ fue mayor que en hombres $(4,6 \%)$ $\left(\chi^{2}=7,602 ; p=0,006\right)$. Esta asociación se mantuvo tras realizar ajuste por edad, IMC, hábitat y tabaquismo mediante regresión logística binaria 
(tabla 2). La cifra de TSH fue mayor también en mujeres $\left(2,42\right.$ vs $\left.2,06 \mu \mathrm{U} / \mathrm{ml} ; U_{M W}=11146 ; p=0,003\right)$.

\section{DISCUSIÓN}

Antes de iniciar la discusión de nuestros resultados hemos de advertir que los participantes fueron reclutados en las propias consultas, por lo que es posible que no sean exactos representantes de la población general, aunque, a la vista de la utilización de nuestras consultas, pensamos que poca diferencia debe existir. De igual forma, la cifra de prevalencia global puede estar ligeramente sesgada al alta por la mayor presencia de mujeres (casi $54 \%$ ) en nuestra muestra.

La prevalencia de hipotiroidismo subclínico obtenida en nuestro estudio $(9,3 \%)$ es inferior a la encontrada en dos trabajos realizados en pacientes con obesidad 'mórbida' (IMC >40)11,12, que hallan cifras en torno al $14 \%$ (uno de ellos referido sólo a mujeres), y a otro en pacientes obesos que refiere una prevalencia del $11 \%{ }^{13}$. Estos porcentajes, en cualquier caso, no difieren demasiado de las halladas en otros estudios realizados en población general, que oscilan entre el 3 y el 15\%14,15. Aunque al considerar la prevalencia de la patología tiroidea hemos de tener en cuenta factores ambientales, geográficos y étnicos responsables de variaciones poblacionales, nuestra cifra es casi idéntica a la encontrada en el mayor estudio realizado hasta la fecha (Colorado Thyroid Disease Prevalence Study ${ }^{16}$, que alcanza el $9,0 \%$ en una muestra de más de 25.000 personas.

No hemos encontrado tampoco una correlación estadística entre los niveles de IMC y los valores séricos de TSH en nuestra muestra de pacientes obesos y sobrepesos, lo que concuerda con los datos obtenidos en la mayoría de los estudios realizados hasta el momento ${ }^{1,11,12,17-19}$ y contradice a otros que sí la han observado en sus series ${ }^{5,6,20}$. Parece por tanto que el hipotiroidismo produce claramente aumento de peso, pero la influencia que la obesidad pueda tener sobre la función tiroidea es aún poco clara y tendremos que esperar a nuevos y definitivos estudios para conocer si existe o no realmente. En la práctica, ello significa que en el estudio de una obesidad debemos solicitar TSH y T4 libre para descartar su origen tiroideo, pero no sería necesario monitorizar estos parámetros en el seguimiento de la obesidad.

En nuestro trabajo no hemos podido demostrar un aumento de la tasa de hipotiroidismo a medida que aumenta la edad de la población analizada, aun- que existen algunos estudios que apoyan esta relación ${ }^{14,15,21}$. Sí en cambio queda patente la mayor frecuencia de hipotiroidismo subclínico en mujeres, ya destacado en numerosos trabajos ${ }^{10,14,15}$, con una relación mujer/hombre entre 2-8/1 según la revisión de Garcia y cols ${ }^{22}$. Incluso hemos encontrado que los niveles medios de TSH en plasma son superiores en este colectivo, aún dentro de rangos considerados normales.

Nos ha llamado la atención la diferencia encontrada entre los valores de TSH en medio urbano y rural, que pensamos pueden ser debidas a características medioambientales derivadas de la mayor o menor presencia de yodo en las aguas de abastecimiento, aunque no hemos podido contrastar esta sospecha. En cualquier caso, es posible que este factor deba ser tomado en consideración a la hora de explicar diferencias en la prevalencia de hipotiroidismo ${ }^{23}$.

Finalmente, no hemos podido encontrar en nuestra muestra de obesos y sobrepesos la relación entre los niveles de hormonas tiroideas con el hábito tabáquico apuntada por algunos autores ${ }^{24,25}$. El tiocianato, uno de los principales tóxicos del tabaco, se ha relacionado con la inhibición de la síntesis de hormonas tiroideas e inducción de hipotiroidismo subclínico ${ }^{26}$. Por el contrario, otros estudios han encontrado menor frecuencia de hipotiroidismo en fumadores ${ }^{27,28}$, lo que habla a favor de un posible efecto dual, en función del estado tiroideo previo, que mencionan algunos autores ${ }^{26}$.

En resumen, la prevalencia de hipotiroidismo subclínico en la población que sufre de exceso de peso, bien obesidad o sobrepeso, es similar a la de la población general, siendo esta prevalencia superior en las mujeres. Si bien en nuestro estudio no hemos podido demostrar una correlación entre los niveles séricos de TSH y el IMC, la existencia de estudios que apoyan ambas hipótesis mantiene la controversia respecto a esta relación y hace preciso la realización de nuevos estudios que apoyen una u otra teoría.

\section{BIBLIOGRAFÍA}

1. Fox CS, Pencina MJ, D’Agostino RB, Murabito JM, Seely W, Pearce EN et al. Relations of Thyroid Function to Body Weight. Arch Intern Med. 2008; 168(6):587-5.

2. Gopinath B, Liew G, Flood VM, Wang JJ, Kifley A, Leeder $\mathrm{SR}$ et al. The association between weight gain and thyroid function in an older population. Arch Intern Med. 2008; 168(20):2283-4.

3. Knudsen N, Laurberg P, Rasmussen LB, Bülow I, Perrild H, Ovesen $L$ et al. Small Differences in Thyroid Function May Be Important for Body Mass Index and the Occurrence of Obesity in the Population. J Clin Endocrinol Metab. 2005; 90:4019-24. 
4. Ortega E, Pannacciulli N, Bogardus C, Krakoff J. Plasma concentrations of free triiodothyronine predicts weight change in euthyroid persons. Am J Clin Nutr. 2007; 85(2):440-5.

5. Kumar H, Verma A, Muthukrishnam J, Modi KD. Obesity and hypothyroidism: symbiotic coexistence. Arch Intern Med. 2008; 168(19):2168

6. Malavazos A, Cereda E, Delnevo A, Passeri E, Tufano A, Sburlati $L$ et al. Thyroid function and body weight: should consider the interplay with insulin resistance and fat distribution. Arch Intern Med. 2008; 168(20):2284-85.

7. Instituto Nacional de Estadística. Encuesta Nacional de Salud 2006 (13 de marzo de 2008). Disponible en http://www. ine.es [consultado el 06-09-10].

8. Flegal KM, Carroll MD, Ogden CL, Curtin LR. Prevalence and Trends in Obesity Among US Adults, 1999-2008. JAMA. 2010; 303(3):235-41.

9. Wilson G, Curry RW. Subclinical Thyroid Disease. Am Fam Physician. 2005; 72:1517-24.

10. Fatourechi V. Subclinical Hypothyroidism: An Update for Primary Care Physicians. Mayo Clin Proc. 2009; 84(1):6571.

11. Rotondi M, Leporati P, La Manna A, Pirali B, Mondello T, Fonte $\mathrm{R}$ et al. Raised serum TSH levels in patients with morbid obesity: is it enough to diagnose subclinical hypothyroidism? Eur J Endocr. 2009; 160:403-8.

12. Navarro Rodriguez MC, Saavedra Santana P, De Pablos Velasco P, Sablón González N, De Miguel Ruiz E, Castro Medina $\mathrm{R}$ et al. Estilo de vida, nivel socioeconómico y morbilidad en mujeres posmenopáusicas con obesidad de grados II y III. Endocrinol Nutr. 2009; 56(5):227-32.

13. Verma A, Jayaraman M, Kumar HK, Modi KD. Hypothyroidism and obesity. Cause or effect? Saudi Med J. 2008; 29(8):1135-8

14. Torné Coll M, Azagra Ledesma R, Espina Castilla A, Vargas Vilardosa E, Reyes Camps A. Hipotiroidismo subclínico en la consulta de Atención Primaria. Aten Primaria. 2006; 37(3):175-76.

15. Díez JJ. Hipotiroidismo subclínico. Endocrinol Nutr. 2005; 52(5):251-9.
16. Canaris GJ, Manowitz NR, Mayor GM, Ridgway EC. The Colorado thyroid disease prevalence study. Arch Intern Med. 2000; 160:526-34.

17. Sang Shon H, Dal Jung E, Hee Kim S, Hyun Lee J. Free T4 is negatively correlated with body mass index in euthyroid women. Korean J Intern Med. 2008; 23(2):53-57.

18. Alevizaki M, Saltiki K, Voidonikola P, Mantzou E, Papamichael C, Stamatelopoulos K. Free thyroxine is an independent predictor of subcutaneous fat in euthyroid individuals. Eur J Endocrinol. 2009; 161:459-65.

19. Reinehr T, Andler W. Thyroid hormones before and after weight loss in obesity. Arch Dis Child. 2002; 87:320-3.

20. Weiss RE, Brown RL. Doctor...Could it be my thyroid? Arch Intern Med. 2008; 168(6):568-9.

21. Sender Palacios MJ, Vernet Vernet M, Pérez López S, Faro Colomés M, Rojas Blanc M, Pallisa Gabriel L. Enfermedad funcional tiroidea en la población de edad avanzada. Aten Primaria. 2004; 34(4):192-7.

22. García García-Doncel L, Sillero Sánchez A, Aguilar Diosdado M. Hipotiroidismo subclínico. Jano. 2002; 52:270-76.

23. Delange $F$. The disorders induced by iodine deficiency. Thyroid. 1994; 4(1):107-28

24. Vestergaard P, Rejnmark L, Weeke J, Hoeck HC, Nielsen $\mathrm{HK}$, Rungby J et al. Smoking as a risk factor for Graves disease, toxic nodular goiter, and autoinmune hypothyroidism. Thyroid. 2002; 12(1):69-75.

25. Utiger RD. Cigarette smoking and the thyroid. N Engl $J$ Med. 1995; 333(15):1001-2.

26. Tziomalos K, Charsoulis F. Endocrine effects of tobacco smoking. Clinical Endocrinol (Oxf). 2004; 61:664-74.

27. Schlienger JL, Grunenberger F, Vinzio S, Goichot B. Smoking and the thyroid. Ann Endocrinol (Paris). 2003; 64(4):309-15

28. Asvold $B$, Bjorg $T$, Nilsen $T$ et al. Tobacco smoking and Thyroid Function: A population Based-Study. Arch Intern Med. 2007; 167(13):1428-32. 